\title{
LOS ACTOS UNILATERALES DE LOS ESTADOS EN DERECHO INTERNACIONAL: LOS TRABAJOS DE CODIFICACIÓN EN LA COMISIÓN DE DERECHO INTERNACIONAL
}

\author{
Nicolás GUERRERO PENICHE* \\ Víctor RODRÍGUEZ CEDEÑO**
}

RESUMEN: El relator especial sobre los actos unilaterales de los Estados acaba de entregar su quinto informe sobre el tema. Ante ello, y la perspectiva de un nuevo quinquenio que se inicia para los trabajos de la CDI, resulta interesante analizar detalladamente los avances logrados, y los problemas que se presentan para sistematizar su estudio y avanzar en su codificación. El presente artículo tiene por objeto realizar un estudio general de los actos unilaterales de los Estados, vistos éstos desde el enfoque propuesto por el relator especial. Así, los autores analizan principalmente lo relacionado con la definición, clasificación, condiciones de validez y causales de nulidad e interpretación de los actos unilaterales de los Estados.

ABSTRACT: The Special Rapporteur on Unilateral Acts of States has finished his fifth report on this issue. This fact and the upcoming beginning of a new quinquennium of work of the ILC make interesting to analyse in detail the progress achieved and the problems faced in the study and codification of this issue. The aim of this article is to give a general study of the unilateral acts of States from the perspective of the Special Rapporteur. Thus, the analysis of the authors focuses mainly on the definition, classification, invalidity conditions, and interpretation of the unilateral acts of States.

RÉSUMÉ: Le Rapporteur spécial sur les actes unilatéraux des Etats vient de présenter son Cinquième Rapport sur ce sujet. Faisant suite à cette présentation et devant la perspective d'un nouveau quinquennat qui commence en ce qui concerne les travaux de la CDI, il résulte intéressant d'analyser en détail les progrès obtenus et les problèmes qui se présentent pour la systématisation de leur étude et pour avancer dans leur codification. Cet article a pour objet l'étude en général des actes unilatéraux des Etats, sous l'optique proposée par le Rapporteur Spécial. Ainsi, les auteurs analysent principalement ce qui concerne la définition, la classification, les conditions de validité et les causes de nullité et d'interprétation des actes unilatéraux des Etats.

* Maestro en derecho internacional. Asistente de investigación del relator especial de la Comisión de Derecho Internacional (CDI): Víctor Rodríguez Cedeño. Consultor de la UNCTAD en el Programa de Solución de Controversias en Materia de Comercio, Inversión y Propiedad Intelectual.

** Embajador del Servicio Exterior de Venezuela, miembro y relator especial de la CDI sobre el tema de los actos unilaterales del Estado. 


\section{SUMARIO: I. Introducción. II. Delimitación del estudio; elementos} constitutivos de una definición operativa. III. Tipificación de los actos unilaterales. IV. Condiciones de validez y causales de nulidad. V. Sobre la interpretación de los actos unilaterales.

VI. Conclusiones. VII. Anexo.

\section{INTRODUCCIÓN}

La asunción de obligaciones internacionales por parte de los Estados es uno de los ámbitos más importantes del derecho internacional. Si bien existen variadas formas por las que un Estado puede asumir obligaciones, el estudio del tema se ha centrado en los tratados y en la costumbre, fuentes principales del derecho internacional según queda reflejado en el artículo 38 del Estatuto de la Corte que las enumera. Sin embargo, también existen otras modalidades menos estudiadas y definidas, como el tema que nos ocupa: los actos unilaterales.

Los actos unilaterales han sido objeto de examen por la doctrina internacional desde hace varias décadas; uno de los primeros trabajos sobre el tema fue de Anzilotti en 1929; ${ }^{1}$ desde entonces, los estudios de autores de renombre como Pfluger, Venturini, Biscottini, Degan, Suy, Guggenheim, Cahier, y de hispanoamericanos como Barberis, Villagrán Krammer y Miaja de la Muela reflejan la importancia y la atención que se le ha prestado al tema. ${ }^{2}$

Por otra parte, los tribunales internacionales han también considerado algunos de esos actos y comportamientos unilaterales del Estado; inicialmente en la decisión sobre Groenlandia Oriental, por la Corte Permanente

1 Anzilotti, Cours de droit international, París, Sirey, 1929.

2 Pfluger, F., Die einseitigen Rechtsgeschäfte im Völkerrecht; 1936; Venturini, "La portée et les effets juridiques des attitudes et des actes unilatéraux en droit international public", RCADI, vol. 112, 1964-II, pp. 363-467; Biscottini, G., Contributo alla Teoria degli Atti Unilaterali nel Diritto Internazionale, 1951; Degan, "Acte et norme en droit international public", RCADI, vol. 227, 1991II, pp. 357-418; Degan, "Unilateral Acts as Source of Particular International Law”, FYIL, vol. 5, 1994, pp. 149-266; Suy, E., Les Actes juridiques unilatéraux en droit international public, París, LGDJ, 1962, 290 pp.; Guggenheim, "La validité et la nullité des actes juridiques internationaux", RCADI, vol. 74, 1949-1, pp. 191-268; Cahier, "Le comportement des États comme source de droits et d'obligations", Recueil d'études de droit international en hommage à Paul Guggenheim, Genève, IUHEI, 1968, pp. 237-265; Barberis, "Los actos jurídicos unilaterales como fuente de derecho internacional público", Hacia un nuevo orden internacional y europeo: Estudios en homenaje al profesor don Manuel Diez de Velasco, Madrid, Tecnos, 1963, pp. 101-116; Villagran Kramer, "Les Actes unilatéraux dans le cadre de la jurisprudence internationale", Le Droit International à l'aube du XIXe siècle: Réflexions de codificateurs, Nueva York, UN, 1997, pp. 137-161; Miaja de la Muela, "Los actos unilaterales en las relaciones internacionales", REDI, vol. 19, 1967, pp. 429-464. 
de Justicia Internacional (CPJI) en $1933 .{ }^{3}$ En esa ocasión, la corte concluyó que Noruega no podía, sobre la base de la declaración formulada por el ministro de relaciones exteriores, Ihlen, poner dificultades al ejercicio de la soberanía de Dinamarca sobre el territorio de Groenlandia Oriental. La corte estimó que la declaración Ihlen era vinculante jurídicamente; que constituía una promesa, quizás un reconocimiento, y en cualquier caso era una declaración unilateral, un acto unilateral de naturaleza jurídica.

En el mismo sentido, los actos unilaterales fueron discutidos en las decisiones de la Corte Internacional de Justicia (CIJ) en 1974 sobre los ensayos nucleares, cuando Nueva Zelanda y Australia solicitaron a la corte que declarara que los ensayos nucleares que realizaba Francia en el Pacífico eran contrarios al derecho internacional y por ende debían cesar. La corte concluyó entonces que las declaraciones de las autoridades francesas eran vinculantes jurídicamente y que no había dudas en cuanto a la capacidad de estas personalidades para obligar o comprometer al Estado en sus relaciones internacionales. ${ }^{4}$

Esta decisión, que se refiere a uno de los actos unilaterales materiales, la promesa, ha facilitado el estudio del tema, pues la corte reconoció que las declaraciones unilaterales pueden comprometer jurídicamente al Estado, que pueden ser oponibles al Estado autor, además de que su forma no es determinante, pues son manifestaciones de voluntad que deben ser claras, con objeto preciso y hechas públicas, que se les otorgue notoriedad. ${ }^{5}$

Otro caso significativo es el de las actividades militares y paramilitares, en el que la CIJ reconoció que los Estados son libres de contraer obligaciones unilaterales sin condiciones ni límites de duración, y con condiciones y reservas. La corte precisó en este caso que el carácter unilateral no le permite al Estado modificarlas unilateralmente. ${ }^{6}$ Debe considerarse también la decisión sobre la controversia territorial entre Burkina Faso y Mali, en el que se analiza el elemento de intención para determinar el carácter jurídico del acto o de la declaración del representante del Estado. ${ }^{7}$

3 Legal Status of Eastern Greenland (Denmark v. Norway), Reports PCIJ 1933, series A/B, núm. 53.

4 Caso relativo a los ensayos nucleares (Australia $v$. Francia; Nueva Zelandia $v$. Francia), CIJ Reports 1974, pp. 253-457.

5 Párrafos 42 a 60 en el caso de Australia, y 45 a 63 en el caso de Nueva Zelandia, en idem.

6 Caso relativo a las actividades militares y paramilitares en Nicaragua y contra Nicaragua (Nicaragua $v$. Estados Unidos de América), CIJ Reportes 1986, p. 14.

7 Caso relativo a la controversia fronteriza (Burkina Faso v. Mali), CIJ Reportes 1986, p. 554. 
A pesar del abundante estudio doctrinal del tema, y de su consideración por los tribunales internacionales, el examen del tema de los actos unilaterales ha demostrado ser complejo y su codificación suscita controversia.

La Comisión de Derecho Internacional (CDI), en el marco de su labor de codificación y de desarrollo progresivo del derecho internacional, ha intentado elaborar una definición y desarrollar las reglas aplicables a las distintas categorías de actos unilaterales. A comienzos de la década de los setenta, la CDI consideró que el tema era susceptible de ser estudiado, al tiempo que reconocía la dificultad de la empresa. En ese entonces, la CDI consideró que un análisis podría "...proporcionar cierto esclarecimiento autorizado...". Sin embargo, no fue sino hasta 1997 que se consideró a los actos unilaterales como uno de los temas "idóneos para su codificación y desarrollo progresivo". La comisión dijo que el objetivo del examen sería "...tratar de precisar el funcionamiento de esta clase de actos y cuáles son sus consecuencias jurídicas, presentando una exposición del derecho aplicable".

Dada la importancia de los trabajos en el marco de la CDI para la codificación y desarrollo progresivo del tema, resulta indispensable hacer un balance de éstos, señalando las dificultades que se han encontrado, y las soluciones adoptadas, de forma tal que trasluzcan los avances y aportes para el estudio de los actos unilaterales.

\section{DELIMITACIÓN DEL ESTUDIO; ELEMENTOS CONSTITUTIVOS DE UNA DEFINICIÓN OPERATIVA}

Tal como lo señala el relator especial, aunque el tema ha sido objeto de estudio por la doctrina y de consideración judicial, no se le ha dado un tratamiento similar en todos los casos. De hecho, al examinar los trabajos doctrinales, se observa que en la mayoría de los casos se le ha dado un enfoque diferente, lo que, sin lugar a dudas, complica su estudio. Asimismo, las decisiones de ambas cortes presentan divergencias en este tema; mientras que en el caso de Groenlandia Oriental y en el de los ensayos nucleares se consideró que los actos unilaterales referidos eran vinculantes; en el diferendo fronterizo y en el de las actividades militares y paramilitares, la corte concluyó que los actos en cuestión eran políticos y desprovistos de fuerza jurídica. ${ }^{8}$ 
Por su parte, la CDI, tomando en cuenta la importancia de los actos unilaterales, su existencia y su frecuencia cada vez mayor en las relaciones internacionales, y con el fin de fortalecer la seguridad jurídica, incluyó este tema en su agenda en 1996. ${ }^{9}$ La comisión consideró que el tema estaba listo para iniciar su codificación y desarrollo progresivo, con la intención de elaborar reglas específicas que regularan su funcionamiento. ${ }^{10}$ $\mathrm{Al}$ año siguiente, se creó un grupo de trabajo, ${ }^{11}$ y se designó a un relator especial.

Una de las primeras cuestiones a las que dirigió sus esfuerzos el relator especial, fue la de establecer una definición de acto unilateral, tarea que resultó harto compleja y llena de problemas de forma y fondo. Es por ello que el primer informe del relator especial fue casi enteramente consagrado a esta cuestión. ${ }^{12}$ En él, se buscaba determinar la existencia de actos estatales de carácter unilateral que producen efectos jurídicos en el plano internacional; en especial aquellos a los que no se les aplican las normas sobre el derecho de los tratados, recogidas en general en la Convención de Viena de 1969, y que, en consecuencia, merecían la elaboración de reglas específicas para regular su funcionamiento.

Uno de los principales argumentos utilizados para negar la existencia de los actos unilaterales, es que estos actos siempre resultan o se traducen en una relación bilateral. Si bien esto es cierto, pues no hay acto unilateral que no produzca una relación bilateral, lo que caracteriza a este tipo de actos es la unilateralidad en su elaboración, su aplicación, y en relación con los efectos jurídicos que produce, y no su materialización que finalmente se ubica en una relación bilateral, aunque no convencional. ${ }^{13}$ Este

ensayos nucleares (Nueva Zelandia $v$. Francia), cit., nota 4, párrafos 35-53, p. 457. Caso relativo a la controversia fronteriza, cit., nota 7, párrafos 38-40, p. 554. Caso relativo a las actividades militares y paramilitares, cit., nota 6 , párrafos $167-171$ y 257-262, p. 14.

9 El tema de los actos unilaterales fue abordado por la comisión en su 48o. Periodo de Sesiones. Documentos Oficiales de la Asamblea General, quincuagésimo primero periodo de sesiones, suplemento núm. 10 (A/51/10), párrafo 249.

10 Véase "Annex II, Addendum 3. Unilateral Acts of States", Report of the Commission to the General Assembly on the Work of its forty-eighth session, YILC, 1996, vol. II, Part Two, pp. 141143. Donde se propuso un programa general para abordar el estudio del tema.

11 Documentos Oficiales de la Asamblea General, quincuagésimo segundo periodo de sesiones, suplemento núm. 10 (A/52/10), párrafos 8 c) y 193.

12 A/CN.4/486.

13 Esta fue una de las características esenciales de los actos unilaterales que señaló el Grupo de Trabajo. Véase el Informe del Grupo de Trabajo, en el "Capítulo IX. Actos unilaterales" del Informe de la Comisión de Derecho Internacional a la Asamblea General sobre el trabajo en su cuadragésimo noveno periodo de sesiones, 1997. 
enfoque, propuesto por el relator especial y adoptado por al comisión, nos parece el adecuado para tratar el tema.

Otro de los puntos que se abordó al inicio, fue el relativo a la delimitación del tema. Aunque no hay dudas de que los Estados formulan constantemente actos unilaterales, únicamente algunos producen efectos jurídicos, parte de éstos producen efectos jurídicos en el plano internacional. Lo que redujo más el ámbito de estudio, es el hecho de que algunos de estos actos se ubican dentro de la esfera convencional, es decir, que otros de ellos pueden colocarse fuera de esa relación, y son exactamente estos últimos el tema de estudio desarrollado en los trabajos de la comisión.

Para llegar a una definición de acto unilateral, el relator especial procedió, primero, a delimitar el tema, identificando aquellos actos que debían ser excluidos, y luego inició la búsqueda de los elementos constitutivos de la definición.

En primer lugar, decidió referirse a los actos unilaterales en un sentido estricto. A los actos emanados de un sujeto de derecho internacional o de varios de ellos, pero que de alguna manera se colocan en el mismo lado de la norma jurídica, lo que permite afirmar que hay actos unilaterales individuales y colectivos. Por este medio, excluyó todos aquellos actos en cuya elaboración participan dos o más Estados en una relación bilateral o multilateral, convencional.

En segundo lugar, se excluyeron los actos de las organizaciones internacionales, no por que ellos no tengan relevancia, si no por que rebasaba el mandato que le había dado la Asamblea General a la comisión, y por que el examen conjunto de tales actos complicaría el estudio. ${ }^{14}$ Sin embargo, no se obvia la referencia a las organizaciones internacionales. Si bien se excluye su participación en la elaboración de estos actos, se les considera en cuanto a los efectos de los actos emanados de los Estados, es decir, en condición de destinatario y no como autor de tales actos. Siguiendo esta línea de argumentación, resulta interesante constatar que queda abierta la posibilidad de que los actos elaborados por un Estado puedan estar dirigidos a otros sujetos de derecho internacional.

De la misma forma se separaron, y no fue fácil, como demuestra la larga discusión en la comisión en 1998,15 los actos políticos de aquellos

14 Sobre los actos unilaterales de las organizaciones internacionales, véase Viraly, "Les Actes unilatéraux des organisations internationales", Le Droit International à l'aube du XIXe siècle: Réflexions de codificateurs, Nueva York, UN, 1997, p. 163.

15 Véase las sesiones 2524a a 2527a del 5 al 8 de mayo de 1998. 
que tienen consecuencias jurídicas. El problema consistía en determinar cuándo se está ante un acto político, y cuándo ante un acto de naturaleza jurídica. La cuestión es aun más difícil si se considera que nada parece impedir que un acto formalmente político tenga efectos jurídicos.

La conclusión a la que se ha llegado en la comisión, que nos parece, por lo demás atinada, es que la naturaleza del acto y la fundamentación de su obligatoriedad se basan en la intención del Estado; intención que puede ser expresa, pero también puede ser inferida, como lo determinó la corte en el Caso de los Ensayos Nucleares, en cuanto a las declaraciones formuladas por autoridades francesas, como las del presidente de la república y el ministro de relaciones exteriores. ${ }^{16}$

Asimismo, se dejaron de lado los actos jurídicos unilaterales del Estado que no pueden producir efectos en el ámbito internacional. Como de la Ley Helms Burton que si bien es unilateral, interna e incuestionablemente jurídica, no puede surtir efectos en el plano internacional.

Se separaron también todos aquellos actos que entran en la esfera convencional y a los cuales se les aplican las reglas relacionadas con la materia, en particular, la Convención de Viena sobre el Derecho de los Tratados, de 1969. Tales son los actos unilaterales consabidos, fáciles de identificar y de ubicar en la relación convencional, como serían la firma, la ratificación, el depósito e incluso las reservas y las declaraciones interpretativas, que son actos unilaterales y que la comisión examina por separado, sobre la base del informe preparado por Alain Pellet. ${ }^{17}$

Desde el inicio del examen del tema se decidió dejar de lado los comportamientos y las actitudes del Estado que si bien producen o pueden producir efectos jurídicos, no pueden ser fácilmente definidos como actos jurídicos en el sentido estricto del término. Si bien su valor e importancia son innegables, como demuestra su consideración por los tribunales internacionales, ${ }^{18}$ el silencio no es un acto en un sentido estricto. ${ }^{19}$ Es un comportamiento que puede producir efectos jurídicos.

16 Caso relativo a los ensayos nucleares.., cit., nota 4.

17 Véase sus informes presentados ante la comisión sobre las reservas a los tratados.

18 En el caso de la sentencia del rey de España, la corte estableció que Nicaragua no podía invocar la nulidad del fallo, por que no protestó y reconoció por su conducta, la validez del fallo, Caso relativo al laudo arbitral emitido por el rey de España (Honduras v. Nicaragua), CIJ Reportes 1960, p. 192.

19 Véase la discusión sobre este término en el Tercer Infome sobre los Actos Unilaterales del Estado, A/CN.4/505, p. 8. 
Se descartó del estudio porque el silencio es siempre un comportamiento reactivo, relacionado con un acto $\mathrm{u}$ otro comportamiento preexistente, lo que le ubica en la relación convencional. Por medio del silencio, un Estado puede adquirir derechos y contraer obligaciones; por ejemplo puede aceptar una oferta: qui tacet consentire videtur; puede expresar la voluntad de reconocer como legítimo un estado determinado de $\operatorname{cosas},{ }^{20} \mathrm{O}$ puede expresar su oposición a una situación: qui tacet negat.

Como se señaló en la comisión, el acto unilateral está también íntimamente relacionado con la responsabilidad internacional, con el estoppel y con la formación de la costumbre. Aunque inicialmente se le pidió al relator especial que considerará esta relación, finalmente se concluyó que si bien tenían alguna relación, ellos no constituían actos unilaterales en el sentido señalado.

En el caso de la responsabilidad internacional, tema que estudió la comisión, sobre la base de los trabajos de James Crawford, hay una relación con el tema de los actos unilaterales. En efecto, el acto por el cual un Estado viola un compromiso internacional es, por lo general, aunque no exclusivamente, un acto unilateral. También, un Estado puede ser responsable internacionalmente por la violación de un acto unilateral que le es imputable y que ha sido formulado conforme a las exigencias de validez establecidas, tema que fue ampliamente abordado en el quinto informe sobre actos unilaterales. ${ }^{21}$ Pero, estos actos no se asimilan necesariamente a los actos unilaterales que interesan a la comisión.

El tema de los actos unilaterales también está relacionado con el estoppel, una figura esencialmente procesal de origen anglosajón que ha sido considerado por la corte en varios casos, como el de los Empréstitos Serbios, el Templo de Préah Vihéar y el de la Mar del Norte. ${ }^{22}$ Si bien el acto que puede dar lugar al recurso de estoppel es un acto unilateral, no es exactamente el acto bajo estudio. Los actos y comportamientos relacionados con el estoppel no tienen como objetivo preciso crear una obligación jurídica a cargo del Estado que lo realiza, pues el elemento característico del estoppel no es la voluntad del Estado, sino la expectativa que se crea en otro Estado.

20 Véase Anzilotti, op. cit., nota 1, p. 535.

21 A este respecto, véase los informes preparados por el relator especial James Crawford.

22 Case concerning the payment of various Serbian Loans issued in France, PCIJ series A, núms. 20/21, pp. 37-39. Caso relativo al Templo de Preah Vihar (Cambodia v. Tailandia), CIJ Reports 1962, pp 27-33. Casos relativos a la Plataforma Continental del Mar del Norte, CIJ Reportes 1969 , p. 3. 
Sin embargo, la conducta que da lugar al estoppel puede involucrar un acto positivo o uno pasivo, como el silencio. Además, en la figura del estoppel, no es necesario que la conducta original se lleve a cabo con la intención de crear obligaciones jurídicas, lo que es una característica esencial de los actos unilaterales que nos ocupa.

En el mismo sentido, no se puede ignorar que los actos de formación de la costumbre son por lo general actos unilaterales, pero ellos tampoco responden exactamente a la categoría de actos analizados. El proceso consuetudinario para la formación de la costumbre reúne, indispensablemente, dos elementos: el cumplimiento repetido de los actos, denominado consuetudo, y la convicción de los sujetos autores de que el cumplimiento de tales actos es obligatorio conforme al derecho, designado opinio juris sive necessitatis.

Por medio de esta figura, los Estados participan en el proceso de elaboración de normas jurídicas a través de sus comportamientos. Se trata de actos unilaterales desde un punto de vista formal, que son manifestaciones de voluntad que guardan relación con la convicción de que una práctica es derecho. Sin embargo, se debe destacar que la fuente de la costumbre puede provenir también de actos jurídicos convencionales, aunque éstos pudieran ser unilaterales en relación con el proceso de formación de la costumbre. ${ }^{23}$

Estos actos pueden ser excluidos de los actos puramente unilaterales por considerar que surten efectos en una especie de relación convencional tácita, en otras palabras, surten efectos cuando coinciden con otros actos de igual naturaleza, y contribuyen a la formación de una norma consuetudinaria.

También se cuestionó en el seno de la CDI, sobre las declaraciones que formulan los agentes de un Estado en un proceso, y la posibilidad de que sean considerados actos unilaterales. Según el relator, tales declaraciones pueden ser unilaterales en el sentido señalado si resultan autónomas; si mediante ellas se realiza una promesa o un reconocimiento. $\mathrm{Su}$

23 A este respecto, véase Guggenheim, op. cit., nota 2, p. 111, y Rousseau, C., Droit international public, t. I: Introduction et sources, 1970, pp. 334-337. Véanse también los siguientes casos: The S. S. Wimbledon, PCIJ, serie A, núm. 1, pp. 25-28. Case relating to the Territorial Jurisdiction of the International Commission of the River Oder, PCIJ, serie A, núm. 23, p. 27. Caso relativo al derecho de asilo (Colombia-Perú), CIJ Reportes 1950, p. 277. Casos relativos a la Plataforma Continental del Mar del Norte, CIJ Reportes 1969, p. 3. Caso relativo a las actividades militares y paramilitares, op. cit., nota 6, p. 14. 
interpretación, que debe ser restrictiva como lo dijo la misma corte en el caso de $1974,{ }^{24}$ puede determinar si se trata o no de un acto puramente unilateral.

Otra cuestión afecta el estudio emprendido acerca de los actos unilaterales, es su diversidad lo que incide también en la posibilidad de elaborar reglas de aplicación común. No obstante, la comisión llegó a la conclusión de que es posible elaborar reglas comunes en relación con la formulación del acto. Así, por ejemplo, serían reglas de aplicación común, las relacionadas con la definición, la capacidad del Estado, la determinación de las personas habilitadas para actuar en nombre del Estado. Esta posibilidad de elaborar normas generales ha quedado reflejada en los cinco informes presentados por el relator especial ante a la comisión. ${ }^{25}$

En este sentido, en la CDI y en la Sexta Comisión fue objeto de discusión la relación existente entre la Convención de Viena sobre el Derecho de los Tratados y el proyecto de codificación sobre los actos unilaterales. Para unos debía seguirse de cerca la convención sin que ello significara que era aplicable mutatis mutandis; para otros, los trabajos debían separarse de la Convención de Viena por que se trataba de actos muy diferentes. Finalmente, se determinó que no se podía ignorar el texto de la convención, su estructura y su contenido, así como los trabajos preparatorios de la conferencia de 1968-69, sino que el relator debía mantener un paralelismo flexible, considerando la convención como una fuente válida, sin ignorar que los actos unilaterales en cuestión son una categoría de actos muy particulares, distintos a los convencionales.

Después de la separación de los actos unilaterales que no interesan al estudio del tema, debíamos intentar determinar los elementos constitutivos de una definición aceptable y fundamentalmente operativa, como veremos, del acto unilateral que nos interesa, que corresponden a una manifestación de voluntad unilateral en principio, a nuestro juicio, inequívoca, término este último que no fue aceptado por la mayoría de los miembros de la comisión.

En opinión del relator especial, un componente que caracteriza al acto unilateral y que ha provocado una larga discusión en la comisión, es

24 Véase Caso relativo a los ensayos nucleares..., cit., nota 4.

25 Primer Informe, Quincuagésimo periodo de sesiones, 1998, Doc. No. A/CN.4/486. Segundo Informe, Quincuagésimo primer periodo de sesiones, 1999, Doc. No. A/CN.4/500. Tercer Informe, Quincuagésimo segundo periodo de sesiones, 2000, Doc. No. A/CN.4/505. Cuarto Informe, Quincuagésimo tercero periodo de sesiones, 2001, Doc. No. A/CN.4/519. Quinto Informe, Quincuagésimo cuarto periodo de sesiones, 2002, Doc. No. A/CN.4/525 con dos adendos. 
el relacionado con su autonomía, característica ampliamente reconocida por la doctrina. ${ }^{26}$ Cabe resaltar que en los reportes del relator especial se hace referencia a una doble autonomía, una en relación con la elaboración, en el sentido de que la formulación del acto depende solamente de una manifestación de voluntad sea individual o colectiva. ${ }^{27}$

En segundo lugar, el acto es autónomo por que no depende de una norma preexistente; si bien es cierto que todo acto jurídico está vinculado a normas anteriores, a aquellas del derecho internacional; con el requerimiento de autonomía, se excluyen todos aquellos actos que están vinculados a otros regímenes, como el derecho de los tratados.

En la definición que se remitió al comité de redacción no se incluye el término autonomía, para atender la sugerencia de algunos miembros. Sin embargo, en los comentarios deberá explicarse con claridad que estos actos, como lo dice con razón la mayor parte de la doctrina, son independientes o autónomos, por que es precisamente esa cualidad la que les caracteriza como actos unilaterales que interesan al estudio de la comisión.

El relator especial incluye dentro de los elementos constitutivos de la definición de estos actos, la notoriedad, como una característica fundamental. Inicialmente se refiere a la necesidad que el destinatario conozca el acto; un acto secreto no puede producir efectos jurídicos.

El último elemento constitutivo de la definición del acto unilateral que presenta el relator especial, es la producción de efectos jurídicos. En los primeros trabajos sobre el tema se hablaba únicamente de obligaciones, considerando que un Estado no puede adquirir derechos mediante la formulación de un acto unilateral, ya que ello significa que impone obligaciones a otro Estado que no ha participado en su elaboración, lo que es contrario a dos principios claramente establecidos en derecho internacional res inter alios acta y pacta tertii nec nocent nec prosent. La doctrina

26 Al respecto, véase Guggenheim, Traité de Droit International Public, Avec mention de la pratique internationale et suisse, 2a. ed., vol. I, 1967, pp. 273-274; Brownlie, Principles of Public International Law, 4a. ed., 1990, p. 638; y Zemanek, "Unilateral Legal Acts Revisited", en varios autores, International Law; Theory and Practice: Essays in Honour of Eric Suy, La Haya, Nijhoff, p. 212, entre otros.

27 La CIJ hizo referencia a la autonomía de los actos unilaterales en este sentido, argumentando que tales actos pueden surtir efectos jurídicos independientemente de la aceptación del destinatario. Caso relativo a los ensayos nucleares, cit., nota 4, párrafos 42-60 y 45-63. 
es casi unánime en este sentido, y la jurisprudencia coincide en afirmar que un Estado no puede imponer obligaciones a otro Estado sin su consentimiento. $^{28}$

La práctica parece confirmar esta tesis, el relator especial propone como ejemplo la declaración del gobierno de Nicaragua en relación con el Tratado de Delimitación Marítima entre Honduras y Colombia, argumentando que no crea obligaciones ni derechos conforme al principio res inter alios acta. Sin embargo, el relator identifica un caso en los que resultaría posible que un Estado imponga obligaciones mediante la formulación de un acto unilateral formal, las declaraciones de neutralidad.

No obstante, el Estado puede reafirmar derechos o preservarlos mediante un acto unilateral, mientras no imponga obligaciones ni adquiera nuevos derechos por uno de esos actos frente a un tercer Estado, sin su consentimiento.

Después de haber examinado los cuatro primeros informes del relator especial, la comisión decidió remitir al comité de redacción cuatro proyectos de artículos de aplicación general: la definición, la capacidad del Estado, las personas habilitadas para formular un acto unilateral y la confirmación ulterior de un acto formulado sin autorización. En el grupo de trabajo, y en el quinto informe del relator especial, se examinaron más detenidamente dos cuestiones: las condiciones de validez y las causales de nulidad, que como mencionamos anteriormente, se asemejan a aquellas contenidas en las normas de Viena de 1969, y una cuestión que ha resultado compleja y discutida: la nulidad de los actos unilaterales contrarios a una decisión adoptada por el Consejo de Seguridad en el marco del capítulo VII.

\section{TIPIFICACIÓN DE LOS ACTOS UNILATERALES}

Uno de los problemas que se encuentran al intentar estudiar los actos unilaterales es la existencia de una gran diversidad de estos actos. Es cierto que si se concluye que hay una diversidad importante de actos unilaterales, resulta difícil establecer reglas aplicables a todos esos actos. Una parte de la doctrina considera que si bien los actos unilaterales tienen diferencias relevantes en cuanto a su contenido y efectos jurídicos, también

28 Véase Consideraciones sobre un tratado entre terceros Estados que pretenden lesionar la soberanía de Nicaragua, Managua, Ministerio de Relaciones Exteriores de Nicaragua, 1999. 
cuentan con similitudes importantes, particularmente desde el punto de vista formal, lo que puede deducirse del examen de los actos unilaterales materiales clásicos, como la promesa, el reconocimiento, la renuncia, entre otros. ${ }^{29}$

Según el relator especial, el aspecto formal común a todos los actos consiste en una manifestación única de voluntad, de origen individual o colectivo que produce efectos por sí misma. En su opinión, se trata de un grupo de actos de naturaleza jurídica formulados por uno o varios sujetos, que nacen y pueden producir efectos a partir de su formulación, y cuyos efectos jurídicos no resulta condicionados por la aceptación o por la conducta posterior de otro Estado.

La doctrina aborda de manera muy variada la clasificación de los actos unilaterales. ${ }^{30}$ Los autores, en general, agrupan los actos unilaterales desde un punto de vista material, dividiéndolos en el reconocimiento, la protesta, la renuncia y la notificación. Sin embargo, como bien señala el relator especial, esa enumeración confunde la forma y el contenido de los actos. $^{31}$

Algunos autores, como Verdross, recurren al criterio material o de contenido del acto, clasificándolos en negocios jurídicos unilaterales independientes (la notificación, el reconocimiento, la protesta, la renuncia y la promesa), negocios jurídicos internacionales dependientes (ofrecimiento y la aceptación, la reserva y la sumisión a la jurisdicción de la Corte Internacional de Justicia), y negocios jurídicos asociados a determinadas situaciones, mixtos (ocupación, derelicción (sic) y el negotiorum gestio). ${ }^{32}$

29 Los profesores Combacau y Sur señalan a este respecto que "aunque (los actos unilaterales) no se mencionan en el artículo 38 del Estatuto de la Corte, son tan numerosos como diversos, y su función es considerable". Combacau, Jean y Sur, Serge, Droit international public, 4a. ed., París, 2001.

30 Por ejemplo, Pfluger distingue entre actos formales y no formales, habla también de actos unilaterales condicionados, revocables, no condicionados y no revocables y, por otra parte, de los actos unilaterales autónomos y dependientes. Pfluger, F., op. cit., nota 2, p. 64.

31 Combacau, Jean y Sur, Serge, op. cit., nota 29, p. 94.

32 Verdross, Alfred, Derecho internacional, Madrid, 1997, pp. 103 y 104. Sobre la base de un criterio similar se pronuncian otros autores, como Remiro Brotóns, quien los clasifica basándose en su objeto: reconocimiento, renuncia, promesa: Remiro Brotóns, Derecho internacional, Madrid, McGraw-Hill, 1997, p. 175. Además, coincide con Daillier y Pellet, quienes señalan que "una clasificación material es la más fecunda", agregando que "se distinguen, en general, los principales tipos siguientes: la notificación, el reconocimiento, la protesta, la renuncia, la promesa". Daillier y Pellet, Droit international public, París, LGDJ, 1999, p. 358. Por su parte, Venturini, basándose también en el criterio material, distingue la promesa, la renuncia, la revocación, la denuncia y la declaración de guerra. Venturini, "Attitudes et actes unilatéraux des Etats", $R C A D I, 1964$, vol. II, t. 112, pp. 414 y ss. Otros autores abordan la cuestión en forma diferente, lo que refleja las dificultades para establecer un criterio válido, por ejemplo, Skubiszewski presenta una clasificación más general, identificando: el acto como 
Por otra parte, Biscottini presenta una clasificación sobre la base de los efectos jurídicos, distinguiendo: actos en los cuales la voluntad tiene una función independiente de otros hechos y actos en los cuales la voluntad no tiene una función independiente sino que están vinculados a otros hechos. ${ }^{33}$ De forma similar, Suy distingue los actos constitutivos, extintivos, traslativos y declarativos. ${ }^{34}$ En el mismo sentido, Rousseau identifica como actos expresos el acto-condición (la notificación), los actos creadores de obligaciones (promesa y reconocimiento), los actos confirmativos de derechos (protesta) y los actos por los que se abandonan derechos (renuncia). ${ }^{35}$

Otro autor que recurre también al criterio de los efectos jurídicos es Jacqué, quien distingue: los actos que crean obligaciones a cargo de su autor, los actos por los cuales el Estado abandona un derecho y aquellos por los cuales el Estado confirma la existencia de derechos sobre los cuales es titular. ${ }^{36}$ Para el relator especial, resulta de especial interés que este autor hace referencia a dos categorías de actos, aquellos por los cuales se asumen obligaciones, y a los actos mediante los cuales se confirman derechos o posiciones jurídicas; según explica, los actos por los cuales el Estado autor abandona un derecho pueden asimilarse a los primeros pues crea una obligación para su autor.

En este sentido, resulta interesante observar que Rigaldies distingue "entre los actos jurídicos unilaterales susceptibles de crear derechos para terceros, así como obligaciones para su autor, de los actos que son susceptibles de crear obligaciones para terceros", 37 siendo únicamente los primeros estrictamente unilaterales, pues los otros son dependientes de otros actos como la aceptación.

instrumento (declaración y notificación) y el acto desde el punto de vista de su contenido y de sus efectos (reconocimiento, protesta, promesa y renuncia) "Les actes unilatéraux des Etats". Skubiszewski, Droit international: bilan et perspectives, París, Pédone, 1991, p. 235.

33 Biscottini, op. cit., nota 2, pp. 18-24.

34 Suy, E., op. cit., nota 2, p. 42.

35 Rousseau, Charles, op. cit., nota 23, p. 421.

36 Jacque, Jean-Paul, Eléments pour une théorie de l'acte juridique en droit international, París, LGDJ, 1972, p. 336.

37 Citado por Venturini, "El alcance y los efectos jurídicos de las actitudes y de los actos unilaterales de los Estados", RCADI, 1964/II; Rigalides, Francis, "Contribution a l'étude de 1'acte juridique unilatéral en droit international public", op. cit., p. 425 (sic). 
Por su parte, Dupuy, recurriendo al criterio de los efectos, los distingue según permiten “...oponerse a una situación jurídica, al ejercicio de derechos soberanos o a la creación de compromisos jurídicos". ${ }^{38}$

El relator especial, consciente de la necesidad de incluir la opinión de los gobiernos para lograr establecer un criterio válido para fundamentar la clasificación, hace referencia a los comentarios de los gobiernos formulados al responder el cuestionario elaborado por la comisión en 1999 y en la Sexta Comisión de la Asamblea General, que permiten apreciar, igualmente, la diversidad de criterios para basar la clasificación de estos actos.

En especial, hace referencia a la respuesta del gobierno de Italia que los divide en tres categorías: Actos unilaterales que se refieren a la posibilidad de hacer valer una situación jurídica; actos unilaterales que crean obligaciones jurídicas; y actos unilaterales que se requieren para el ejercicio de un derecho soberano. ${ }^{39} \mathrm{~A}$ aquella del gobierno de la Argentina, que por su parte, distinguió "cuatro tipos clásicos de actos unilaterales: la promesa, la renuncia, el reconocimiento y la protesta". ${ }^{40}$

De las opiniones doctrinales y opiniones de los gobiernos en materia de clasificación, el relator especial concluye que aunque puedan ser varios los criterios, existe una gran coincidencia sobre los más importantes actos unilaterales materiales que se consideran clásicos.

Sin embargo, opina que basar una clasificación en los actos materiales presenta varios problemas, en primer lugar, porque no podría afirmarse que no existen otros actos unilaterales distintos a los denominados clásicos. ${ }^{41}$ La diversidad a la que se hace referencia impide una enumeración taxativa de los actos unilaterales desde el punto de vista material, lo que complicaría enormemente la agrupación de reglas.

Resulta lógico pensar que no se puede descartar la posibilidad de que existan otros actos unilaterales distintos a los mencionados, además de que no resulta fácil calificar un acto unilateral de un Estado como uno u otro de los actos enumerados, pues un mismo acto puede ser calificado en forma distinta.

Como ejemplo de la dificultad de clasificación desde un punto de vista material, el relator muestra la diversidad con que la doctrina y la juris-

38 Dupuy, Jean-Marie, Droit international public, París, Dalloz, 1995, p. 267.

39 A/CN.4/511, cap. II. Observaciones generales, respuesta de Italia.

40 Idem, respuestas a la pregunta núm. 4, respuesta de la Argentina.

41 Por ejemplo, además de los actos ya mencionados en las clasificaciones presentadas, Fiedler habla de otros actos que considera unilaterales, como "declaraciones, seguridades, promesas, promesas unilaterales de garantía o promesas-confirmación”. Fiedler, W., op. cit., p. 518 (sic). 
prudencia internacionales, califican a la declaración Ihlen, ya sea como una promesa internacional, ${ }^{42}$ como un reconocimiento o una renuncia, ${ }^{43}$ como un reconocimiento, ${ }^{44}$ como un consentimiento, ${ }^{45}$ y como un promesa. ${ }^{46}$

Según el relator especial, todos los actos mencionados se formulan a través de declaraciones unilaterales, de origen individual o colectivo, declaraciones que están sujetas a formas definidas en cuanto a sus condiciones de validez, reglas aplicables independientemente de su contenido o calificación material. La promesa, la renuncia, el reconocimiento, la protesta o cualquier otro acto es formulado mediante una manifestación de voluntad con la intención de producir efectos jurídicos y son esos efectos los que varían.

Para ello, el relator realiza un extenso análisis de cuatro actos unilaterales materiales: la promesa, la renuncia, el reconocimiento, y la protesta. Con ello pretende demostrar la factibilidad de realizar una clasificación basada en los efectos jurídicos, más que en el aspecto material del acto. Divide estos cuatro actos materiales en dos grupos; en el primero incluye la promesa, la renuncia, y el reconocimiento; y en el segundo la protesta.

Por la promesa el Estado autor asume una obligación; es "el acto jurídico unilateral en virtud del cual un Estado se obliga con respecto a ter-

42 Garner considera la declaración Ihlen como una promesa, aunque agrega que ella debe ser considerada como un elemento constitutivo de un acuerdo. Garner, J., "The binding force of unilateral declarations", AJIL, 1933, p. 493.

43 Por ejemplo, el gobierno de Noruega, en su contramemoria, señala: “...el señor Ihlen no quiso nunca, evidentemente, prometer el asentimiento del Gobierno noruego a esta política de cierre...", Contramemoria del Gobierno de Noruega, p. 556.

44 Careeau, por ejemplo, indica que "el reconocimiento formal por Noruega de las pretensiones danesas sobre Groenlandia impedía en lo sucesivo volver sobre este acto unilateral y negar sus consecuencias jurídicas", Droit International, París, Pédone, 1988, p. 209.

45 Mediante la Declaración Ihlen, Noruega constató la existencia de un hecho con valor jurídico y declaró la voluntad de considerar como legítima la situación jurídica constatada, lo que parece estar reflejado en la decisión de la corte, cuando señala que "si esta era la tesis que el Gobierno danés sostenía antes, después y durante sus demandas a las Potencias, su intención, al dirigirse a las Potencias como lo hizo, debió haber sido ciertamente asegurarse de que las Potencias aceptaban el punto de vista del gobierno danés, a saber que la soberanía se extendía ya a toda Groenlandia, y no convencerles de que consintiesen en que una región de Groenlandia todavía no sometida a la soberanía danesa le quedase sometida en lo sucesivo. El objetivo era asegurarse de que estas Potencias no tratarían ellas mismas de tomar posesión de tal o cual parte no colonizada de Groenlandia. El método seguido para alcanzar este objetivo consiste en inducir a las Potencias a que reconozcan una situación de hecho existente", CIJ Recueil, série A/B, núm. 53, p. 43 y 44. La doctrina también ha examinado la Declaración Ihlen, por ejemplo, Kohen la considera como un ejemplo de consentimiento formal unilateral. Kohen, Possession contestée et souveraineté, París, PUF, 1997, p. 280.

46 Pues, según argumenta el relator especial, mediante esta declaración, Noruega se comprometió a no plantear ninguna dificultad ni de plantear ninguna reivindicación en el futuro, lo que responde a la definición de la promesa como acto unilateral material clásico. 
ceros a un cierto comportamiento"; 47 es una declaración unilateral por la que un Estado se compromete a adoptar un determinado comportamiento respecto de otro Estado o Estados, sin subordinar dicho comportamiento a una contraprestación por parte del beneficiario de la promesa. ${ }^{48}$

La CIJ ha igualmente analizado los efectos jurídicos de la promesa, por ejemplo en los casos de los ensayos nucleares, donde señala que una promesa puede comprometer a su autor a condición que sea pública y que la intención sea clara. La corte precisa que en este caso la promesa implicaba una obligación jurídica, constituía un acto estrictamente unilateral sin contrapartida, aceptación, réplica o reacción de ninguna forma. ${ }^{49} \mathrm{Se}$ concluye, por ende, que a diferencia de otros actos unilaterales "que se refieren a hechos o actos existentes, la promesa origina derechos nuevos en beneficio de terceros". 50

Sin embargo, cuando se trata de actos por los cuales el Estado asume obligaciones unilaterales, deben incluirse otros actos igualmente unilaterales en el sentido que nos interesa. En efecto, el Estado no solamente puede asumir obligaciones unilaterales en relación con uno o más Estados mediante una promesa, ésta es fuente de obligaciones unilaterales, "aunque no es el único acto unilateral que engendra deberes". ${ }^{51}$ A juicio del relator, otros actos materiales tienen un efecto jurídico similar, como serían el reconocimiento o la renuncia.

La renuncia "produce la extinción de dicho derecho, toda vez que no implica su transferencia a otros sujetos", 52 por lo que el Estado abandona un derecho o una pretensión, pero a la vez asume o contrae una obligación. El efecto jurídico que produce el acto unilateral de la renuncia se traduce en la obligación que tiene el Estado de "no impugnar ya los derechos que ha adquirido otro Estado en virtud de su renuncia". ${ }^{53}$

Como señala la doctrina, la renuncia puede ser también convencional; en efecto:

47 Rigaldies, Francis, op. cit., nota 37, p. 426. Verdross, A., op. cit., nota 32, p. 104.

48 Rodríguez Carrión, Alejandro, Lecciones de derecho internacional público, 4a. ed., Madrid, Tecnos, 1998, p. 172.

49 CIJ Recueil 1974, p. 374. Citada en Rigaldies, Francis, op. cit., nota 37, pp. 427 y 428.

50 Daillier y Pellet, op. cit., nota 32, p. 359.

51 Skubiszewski, K., op. cit., nota 32, p. 241.

52 Venturini, G., "El alcance...”, cit., nota 37, p. 414.

53 Cahier, Philippe, op. cit., nota 2, pp. 247 y 248. 
Si la renuncia está estipulada en un tratado, pierde su carácter unilateral ya que su efecto depende de la entrada en vigor del tratado, es decir de la voluntad de la otra parte o partes contratantes. Tampoco la renuncia es unilateral cuando está vinculada a la transferencia de la pretensión, derecho, competencia o facultad abandonados a otro Estado o Estados: en este caso se convierte de nuevo en una transacción contractual. ${ }^{54}$

La renuncia también ha sido objeto de análisis por la jurisprudencia internacional, al respecto cabe recordar el caso en las Zonas Francas de Alta Saboya y de Gex, entre Francia y Suiza, ${ }^{55}$ en el cual Francia reconoció que una renuncia, como acto unilateral en derecho internacional, es obligatoria para el Estado renunciante. ${ }^{56}$

Igualmente, mediante el reconocimiento el Estado puede asumir obligaciones unilaterales a su cargo $;{ }^{57}$ el Estado acepta una situación de hecho, una pretensión jurídica, una competencia o un poder, ${ }^{58}$ y por lo tanto se obliga en algún sentido a asumir una determinada conducta. Por el reconocimiento el Estado autor "constata la existencia de ciertos hechos... o de ciertos actos jurídicos... y admite que pueden oponerse contra el".59

Como reconoce la doctrina, el reconocimiento:

Es el procedimiento en virtud del cual un sujeto de derecho internacional, en particular un Estado, que no ha participado en el nacimiento de una situación o en la iniciación de un acto, acepta que esta situación o este acto se opongan contra él, es decir, admite que se le apliquen las consecuencias jurídicas de uno u otro. El reconocimiento constituye un acto unilateral. ${ }^{60}$

54 Skubiszewski, K., op. cit., nota 32, p. 229.

55 Recueil PCIJ 1932, serie A/B, núm. 46.

56 Rubin, "The international legal effects of unilateral declarations", AJIL, vol. 71, 1977, p. 14.

57 Considerado por la generalidad de los autores como el acto unilateral más importante. Skubiszewski, K., op. cit., nota 32, p. 238; Daillier y Pellet, op. cit., nota 32, p. 358. Verdross y Simma, Universelles Völkerrecht: Theorie und Praxis, Berlín, 1976, p. 427.

58 Skubiszewski, op. cit., nota 32, p. 241.

59 Daillier y Pellet, op. cit., nota 32, p. 358.

60 Ibidem, p. 550. En igual sentido se expresan Jennings y Watts, quienes consideran que "el reconocimiento en un sentido amplio implica la aceptación por un Estado de cualquier hecho o situación que se produzca en sus relaciones con otros Estados... El otorgamiento del reconocimiento es un acto de alcance internacional, que afecta los derechos y obligaciones mutuos de los Estados, y su estatuto o capacidad jurídica en general... El otorgamiento del reconocimiento de un Estado es un acto unilateral que afecta esencialmente las relaciones bilaterales...", Jennings, sir Robert y Watts, sir Arthur (eds.), Oppenheim's International Law, 9a. ed., Londres, Longman, 1992, vol. I, pp. 127,128 y 130. 
Como ejemplo de la práctica estatal en este respecto, el relator señala tres casos: la declaración egipcia del 24 de abril de 1957, por la cual reconoce la validez de la Convención de Constantinopla de 1888 sobre el Canal de Suez; ${ }^{61}$ la declaración de la Comunidad Europea (CE) del 16 de diciembre de 1991 titulada "Directrices sobre el reconocimiento de nuevos Estados en Europa Oriental y en la Unión Soviética", en la que se adoptó una posición común sobre el tema; ${ }^{62}$ y el reconocimiento por Italia de la Declaración de Neutralidad de Malta del 15 de mayo de $1980 .{ }^{63}$

A diferencia de los dos casos anteriores, en el caso de la protesta el Estado no asume obligaciones a su cargo en forma unilateral, sino que interrumpe la formación de un derecho, de un título o de una posición jurídica; el Estado autor intenta reafirmar un derecho al impedir que otro pueda asumirlo.

La doctrina considera la protesta como un acto unilateral material clásico que puede ser realizado mediante un comportamiento o por actos concluyentes; ${ }^{64}$ cuya finalidad un sujeto de derecho manifiesta su intención de no considerar como de derecho un determinado estado de hecho y entiende por ello salvaguardar sus derechos violados o amenazados ${ }^{65}$ y que tiene como efecto jurídico "que el estado de cosas contestado ya no es oponible al Estado que protesta que puede continuar haciendo valer sus propios derechos". .66

En la práctica judicial internacional, el examen de las declaraciones constitutivas de protesta es frecuente, en ese sentido los casos del arbitraje del Chamizal, entre Estados Unidos y México, ${ }^{67}$ el de los decretos de nacionalidad en Túnez y Marruecos ${ }^{68}$ y el caso de los islotes de Minquiers y Ecréhos. ${ }^{69}$

A través del examen anterior, el relator concluye que los actos jurídicos unilaterales pueden agruparse en dos grandes categorías, sobre la base

61 La declaración del gobierno de Egipto fue registrada ante la Secretaría de las Naciones Unidas; Naciones Unidas, Recueil des Traités, vol. 265, p. 299.

62 Véase BYIL, vol. 62, 1991, pp. 559 y 560.

63 International Legal Materials, 1982, p. 396.

64 Pfluger, citado por Suy, op. cit., nota 2, p. 51, habla de comportamientos implícitos directos o indirectos, expresos o tácitos y explícitos e implícitos. Así, se refiere como actos implícitos (de protesta) una demanda ante la corte, la ruptura de relaciones, la declaración de guerra.

65 Suy, op. cit., nota 2, p. 48.

66 Ibidem, p. 80.

67 Sentencia del 15 de junio de 1911, AJIL, vol. 5, 1911, pp. 785-833.

68 Opinión consultiva, Recueil PCIJ 1923, série B, núm. 4.

69 Caso de los islotes de Miniquiers y Ecrehos (Francia v. Reino Unido), CIJ Recueil 1953. 
de sus efectos jurídicos: actos por los cuales el Estado asume obligaciones y actos por los cuales el Estado reafirma un derecho.

\section{CONDICIONES DE VALIDEZ Y CAUSALES DE NULIDAD}

Ya en su sesión del 2000, la cuestión de las condiciones de validez del acto unilateral y sus causales de nulidad, fue examinado por la comisión, con base en el tercer informe presentado por el relator especial, ${ }^{70}$ y que fue posteriormente discutido en el quinto informe del relator especial. ${ }^{71}$

El relator especial señala que las condiciones de validez del acto unilateral son transposables (sic) a aquellas que se plantean en el régimen de Viena en relación con el tratado. ${ }^{72}$ En este sentido, considera que las condiciones de validez del acto unilateral se refieren a la formulación del acto por un Estado, por un representante autorizado o habilitado para actuar en su nombre y comprometerle en el plano internacional, la licitud del objeto, el cual no debe estar en contradicción con una norma imperativa de derecho internacional y la manifestación de la voluntad exenta de vicios.

Resulta pertinente la precaución señalada en el quinto informe, en el sentido de que la inclusión de proyectos de artículos relativos a las causales de nulidad de un acto unilateral no puede significar debilitar el principio que se habilita en este contexto para fundamentar la obligatoriedad de estos actos (acta sunt servanda), equivalente éste al principio pacta sunt servanda, contenido en la convención de 1969. Es precisamente sobre la base del principio acta sunt servanda, que se construye posteriormente el artículo 7o. del proyecto sobre el tema, y que expresa un principio general aplicable a los actos unilaterales en su conjunto.

Como se expuso con anterioridad, el proyecto de artículos de la comisión se limita a analizar la formulación de actos unilaterales por parte del Estado, aunque se reconoce la capacidad de otros sujetos de derecho internacional de formular tales actos. En este sentido, solamente las personas habilitadas pueden actuar en nombre del Estado y comprometerle en sus relaciones internacionales. ${ }^{73}$

70 Doc. A/CN.4/505.

71 Documento que fue introducido en la primera parte del 54o. periodo de sesiones en 2002. Doc. A/CN.4/525/Add.1.

72 A este respecto, véase los artículos 42 a 53, 69 a 71 de la Convención de Viena de 1969.

73 No se plantean dudas en cuanto a la representatividad del jefe de Estado, la CIJ lo afirmó en 
Como señaló el relator especial en su segundo informe, el Estado sólo puede ser comprometido por sus representantes, es decir, aquellas personas que por su investidura u otras circunstancias estarían habilitadas para ello. ${ }^{74} \mathrm{Si}$ bien la determinación de las personas con capacidad para emitir actos unilaterales en nombre del Estado depende tanto del derecho interno como del derecho internacional, se deriva de los trabajos preparatorios de la Convención de Viena, así como de los trabajos y discusiones de la comisión al respecto, que la práctica de los Estados, la doctrina y la jurisprudencia concuerdan en el hecho de que la adquisición de obligaciones es una facultad limitativa, es decir, que se deben tomar en cuenta las facultades expresas de los representantes gubernamentales, aunque la regla general impide la invocación de normas internas para alegar la invalidez de un tratado. ${ }^{75}$

Tal como se plantea en Viena, un acto puede ser nulo cuando se formula en violación de una disposición de derecho interno relativa a la competencia para ello, pero esto sólo podría ser alegado si en dos escenarios distintos: si la violación sea manifiesta, y si afecta una norma de importancia fundamental de derecho interno.

Por otra parte, siempre siguiendo lo establecido en la Convención de Viena, el relator hace referencia a una segunda condición de validez: la licitud del objeto del acto. Al respecto, considera que un acto unilateral contrario a una norma imperativa de derecho internacional, es nulo en forma absoluta. ${ }^{76}$

el Caso de la aplicación de la Convención para la Prevención y Sanción del Delito de Genocidio (Excepciones Preliminares) (Rec. CIJ, 1966, p. 622, párr. 44; ni del jefe de gobierno o del ministro de relaciones exteriores, así lo señaló la Corte Internacional en el Caso de Groenlandia Oriental CPJI. 1933, serie A/B, núm. 53, p. 71, y posteriormente lo confirmó en su decisión de 1974 sobre los ensayos nucleares.

74 Doc. A/CN,4/500, Add.1.p. 79.

75 En su Informe de 1966 a la Asamblea General, la CDI señaló que "when the violation of internal law regarding competence to conclude treaties would be objectively evident to any State dealing with the matter normally and in good faith, the consent to the treaty purported to be given on behalf of the State may be repudiated". Yearbook of the ILC, 1996, vol. II. Report of the ILC on the work of its 18 session, ar. 11, p. 242.

76 La doctrina en general comparte esta opinión, por ejemplo "lorsque... l'acte postérieur est contraire aux normes antérieures, revêtues du caractère de jus cogens, la Cour est obligée de laisser hors application, comme étant absolument nul...", Verzijl, J. H. W., "La validité et la nullité des actes juridiques internationaux", Revue de Droit International, t. XV, 1935, pp. 321-322. En el mismo sentido se expresa Barberis, Julio, op. cit., nota 2, p. 112. 
En el mismo sentido, el relator hace referencia a los vicios de la manifestación de voluntad, asunto que fue abordado en su tercer informe, y que, al igual que en los casos anteriores, sigue paralelamente el derecho de los tratados.

En cuanto a la nulidad de un acto unilateral contrario a una decisión del Consejo de Seguridad, en la comisión se señaló que el artículo 25 de la Carta de las Naciones Unidas imponía la obligación de cumplir las decisiones del Consejo de Seguridad. Además, se sugirió que la norma contenida en el artículo 103 de la carta pudiera aplicarse también a los actos unilaterales, con el fin de que las obligaciones contraídas en virtud de la carta prevalecieran sobre cualquier otra obligación derivada de un tratado o de un acto unilateral. ${ }^{77}$

Por otra parte, en su quinto informe, el relator propuso analizar lo relativo a la pérdida del derecho de un Estado a invocar una causal de nulidad, por su comportamiento, sea implícito o explícito. Referente a la pérdida del derecho de invocar una causal de nulidad, resulta indispensable señalar la referencia que hace la CIJ de esta cuestión en el caso de la sentencia arbitral del rey de España, cuando no reconoció a Nicaragua el derecho de contestar la sentencia arbitral por que había aplicado el tratado que contenía la cláusula de arbitraje. ${ }^{78}$

Sin embargo, el relator especial es de la opinión que la cuestión en el ámbito de los actos unilaterales se plantea en forma diferente, pues habría que distinguir el acto en función de sus efectos jurídicos. Considera que la situación es distinta entre los diferentes actos unilaterales materiales: protesta, reconocimiento, promesa o renuncia. Se interroga sobre la posibilidad de incluir una cláusula de aplicación común a todos los actos unilaterales, argumentando que si bien en los casos de la renuncia y la promesa, el Estado autor podría invocar la nulidad del acto si considera que no se han dado todas las condiciones para que la declaración o acto formulado sea considerado válido, en el caso de la protesta resulta difícil considerar la opción que el Estado autor pueda invocar la nulidad del acto, mientras que ello parecería más factible de parte del Estado destinatario del acto.

78 La corte dijo entonces que: "De l'avis de la Cour, le Nicaragua a, par ses déclarations expresses et par son comportement, reconnu le caractère valable de la sentence et il n'est plus en droit de revenir sur cette reconnaissance pour contester la validité de la sentence". Rec. CIJ, 1966, p. 213. 
Esto lleva al relator a examinar la cuestión de saber si el Estado autor o el que puede invocar la nulidad del acto puede perder el derecho a ello por su comportamiento o por su actitud implícita o explícita. Siguiendo el ejemplo del artículo 45 de la Convención de Viena, responde afirmativamente, argumentando que un Estado que formula un acto por el que adquiere una obligación a su cargo, y actúa expresamente o se comporta de alguna manera que signifique aceptar que tal acto es válido, no podría invocar más adelante la nulidad de la declaración; opinión que queda reflejada en el artículo 5o. de su informe.

\section{SOBRE LA INTERPRETACIÓN DE LOS ACTOS UNILATERALES}

Finalmente, en su quinto informe el relator examina el tema de la interpretación de los actos unilaterales, y propone un artículo de aplicación general para todos los tipos de actos. Ya en su cuarto informe, el relator especial había considerado la posibilidad de que el tema pudiera ser objeto de reglas comunes aplicables a todos los actos unilaterales independientemente de su denominación, su contenido y sus efectos jurídicos.

Uno de los aspectos que ha sido ampliamente discutido en la comisión, sobre la base de la propuesta del relator, es la referencia a las reglas de la Convención de Viena debido a las diferencias fundamentales entre el acto convencional y el acto unilateral. Al respecto, el relator ha considerado que si bien el régimen de Viena es importante, sus disposiciones deben ser adecuadas al carácter particular de los actos unilaterales.

A este respecto, hace referencia a la apreciación que la CIJ hizo de las declaraciones de aceptación de su jurisdicción obligatoria, que aunque considera que pueden no ser "de naturaleza estrictamente unilateral", son una declaración unilateral desde el punto de vista formal y por lo tanto, como lo ha señalado la misma corte, una declaración sui generis. ${ }^{79} \mathrm{La}$ corte señaló a propósito de la interpretación de dichas declaraciones que:

79 Se trata del examen que la CIJ ha hecho de la declaración de aceptación de la jurisdicción al caso de la Competencia en materia de pesquerías (España-Canadá), en cuya decisión preliminar de 1998 señala que: "Una declaración de aceptación de la jurisdicción obligatoria de la corte... constituye un acto unilateral que releva de la soberanía del Estado. Al mismo tiempo, ella establece un vínculo consensual y abre la posibilidad de una relación jurisdiccional con los otros Estados que han hecho una declaración en virtud del parágrafo 2, del artículo 36 del Estatuto y constituye "una oferta permanente de los Estados partes en el Estatuto que no han depositado aún una declaración de aceptación”, Rec. CIJ, 1998, p. 453. 
El régimen que se aplica a la interpretación de las declaraciones hechas en virtud del artículo 36 del Estatuto no es idéntico al que se establece para la interpretación de los tratados en la Convención de Viena sobre el derecho de los tratados [en sus planteamientos declaró que] eso no significaba que las reglas jurídicas y del arte de la interpretación de las declaraciones (y de las reservas) no coincidan con aquellas que rigen la interpretación de los tratados. La corte observa que las disposiciones de la Convención de Viena pueden aplicarse solamente por analogía en la medida en que ellas son compatibles con el carácter sui generis de la aceptación unilateral de la jurisdicción de la corte. ${ }^{80}$

En la opinión del relator especial, la intención del Estado autor debe ser el criterio principal de interpretación y, en consecuencia, se debe hacer mayor hincapié en la labor preparatoria que ofrece una clara indicación de la intención.

Sobre el tema de la interpretación, fue objeto de un amplio acuerdo en la comisión, que la regla general de interpretación del acto unilateral debe basarse en la buena fe y en su conformidad con el sentido ordinario que se atribuye a los términos de la declaración, en su contexto y a la luz de la intención del autor.

Este punto de vista ha sido confirmado por la jurisprudencia internacional, por ejemplo, la CIJ, en el caso de la pesquerías (España/Canadá) al analizar las declaraciones de aceptación de su jurisdicción indicó claramente que interpretaba las palabras pertinentes de la declaración, agregando, de forma natural y razonable, teniendo debidamente en cuenta la intención del Estado interesado, lo que puede deducirse no sólo del texto de la cláusula pertinente, sino también del contexto en que debe leerse dicha cláusula y del examen de los testimonios con respecto a las circunstancias de su preparación y de los objetivos previstos. ${ }^{81}$

Sin embargo, en la opinión del relator, el acto unilateral, por su propia naturaleza, debe ser interpretado en forma diferente al acto convencional. Por una parte, " porque la seguridad jurídica exige retener a título principal la voluntad declarada por el texto... y además... ellos deben interpretarse en forma restrictiva". Las respuestas de algunos gobiernos al cuestionario de la comisión, han confirmado este criterio de interpretación. ${ }^{82}$ Por su parte, la doctrina, por lo general, comparte este criterio; así, 
por ejemplo, Grotius dice que "the measure of correct interpretation is the reference of intent from the most probable indications". ${ }^{83}$

Finalmente, el relator considera que el criterio que priva en el ejercicio de la interpretación de estos actos, debe ser el restrictivo, según lo sostiene la doctrina, lo han afirmado los gobiernos y lo ha señalado la jurisprudencia. Como ejemplo, aunque se refiere solamente a la promesa, la CIJ concluyó en el caso de los ensayos nucleares que "cuando los Estados hacen declaraciones que limitan su libertad de acción futura, una interpretación restrictiva se impone". ${ }^{84}$

Siguiendo la lógica propuesta por el relator, y confirmada por la doctrina y jurisprudencia, el objetivo de la interpretación es determinar la intención del Estado; intención que, según el relator, puede deducirse de la declaración formulada y de otros elementos, como los trabajos preparatorios y las circunstancias en el momento de la formulación del acto. Para ello, el término intención es fundamental; la manifestación de voluntad es la expresión necesaria para la formación del acto, mientras que la intención es el sentido que el autor entiende dar al acto. La intención, sin embargo, no sería suficiente en la determinación del acto, por que ella debe ser del conocimiento del o los destinatarios o que por lo menos ellos hayan tenido la oportunidad de conocerla.

El recurso a los trabajos preparatorios, presentado por el relator especial el año pasado en el proyecto que presentara a la comisión, entonces fue cuestionado por algunos miembros de la comisión quienes consideraron que ello no era posible y que además, serían de difícil acceso si es que se considera que la correspondencia interna de los ministerios de relaciones exteriores o de otras entidades del Estado pueden traducirse en los trabajos preparatorios.

\section{CONCLUSIONES}

Como se ha podido observar, el tema es, sin duda, complejo; son muchas las interrogantes, y muchas las lagunas que existen. Se trata de un ámbito desconocido en el que la práctica no es del todo conocida aunque es constante. El trabajo que se ha realizado en la comisión, si bien limita-

83 Grotius, H., De jure belli ac pacis, Book 2, cap. 16. Cfr. Encyclopedia of Public International Law, vol. II, Amsterdam, Elsevier, 1995, p. 1418.

84 Rec. CIJ, 1974, p. 267. 
do a los actos jurídicos unilaterales de los Estados, representa un interesante avance en el estudio de la materia. La delimitación del estudio a aquellos actos que escapan de una relación convencional, constituye un complemento de las normas establecidas en las convenciones de Viena, incrementando la seguridad jurídica de las relaciones internacionales.

Asimismo, la exclusión de los actos unilaterales emanados de una organización internacional, no sólo facilita el análisis de los actos considerados, sino permitirá que posteriormente, y tal vez sobre la base del proyecto de artículos de los actos de los Estados, se desarrolle otro relativo a aquellos.

La división del proyecto en dos fases, una respectiva a la formulación, y otra, referente a las consecuencias de tales actos, resulta un factor que facilita el estudio de tales actos y el desarrollo de un proyecto de artículos que vaya moldeándose conforme al análisis, y la discusión, tanto en el seno de la comisión, como a partir de las opiniones expresadas por los Estados, y por la doctrina concerniente. La división, ha permitido también mantener la independencia de ambas partes, limitando las dificultades del estudio y codificación relativas a cada sección y no mezclándolas, lo que dificultaría aún más la tarea.

Finalmente, cabe señalar que el proyecto de definición del acto unilateral aprobado por al comisión, y referido al comité de redacción, representa un importante avance para el estudio del tema. No sólo delimita de forma inequívoca los actos bajo estudio, sino que además establece excelentes bases para el estudio de sus consecuencias, pues las obligaciones que se deriven del acto deberán corresponder con la voluntad del Estado emisor.

Si bien, por lo anterior, el análisis del tema en la comisión representa una importante aportación para su compresión y codificación, todavía se está lejos de agotar el tema. Sin duda, los trabajos de codificación y desarrollo progresivo, realizados por el relator y la comisión en su conjunto, se complican por la falta de consenso sobre el tema. Es decir, incluso en el momento de la codificación de los tratados internacionales, la doctrina, la jurisprudencia y la práctica de los Estados, presentaba un alto grado de consenso sobre los límites y el objeto de estudio. Como se ha visto, el caso es oposalmente (sic) distinto para los actos unilaterales; sin embargo, a nuestro parecer, el trabajo conjunto del relator especial, pero en especial su definición y clasificación representan un importante avance en el estudio del tema. 


\section{ANEXO}

\section{TEXTO DE LOS ARTÍCULOS SOBRE ACTOS UNILATERALES DE LOS ESTADOS PRESENTADOS POR EL RELATOR ESPECIAL}

Parte I. Reglas generales

A. De la elaboración del acto unilateral

Artículo 1. Definición del acto unilateral

A los efectos de los presentes artículos, se entiende por acto unilateral del Estado una manifestación de voluntad inequívoca del Estado, formulada con la intención de producir efectos jurídicos en sus relaciones con uno o varios Estados o una o varias organizaciones internacionales y que es del conocimiento de ese Estado o de esa organización internacional.

Artículo 2. De la capacidad del Estado

Todo Estado tiene la capacidad de formular actos unilaterales

Artículo 3. De las personas habilitadas para formular un acto unilateral en nombre del Estado

1. El jefe de Estado, el jefe de gobierno y el Ministro de Relaciones Exteriores son considerados representantes del Estado para formular actos unilaterales en su nombre.

2. También se consideraría que una persona está habilitada para formular actos unilaterales en su nombre si se deduce de la práctica seguida por los Estados interesados o de otras circunstancias, que la intención de esos Estados ha sido considerar a esa persona habilitada para actuar en su nombre para esos efectos.

Artículo 4. De la confirmación del acto unilateral formulado sin autorización

Un acto unilateral formulado por una persona que conforme al artículo 3 no está habilitada para actuar en nombre del Estado, no surtirá efectos jurídicos, a menos que sea confirmado expresamente por ese Estado

Artículo 5. De las condiciones de validez y de la nulidad de los actos unilaterales

1. Error: El Estado [o los Estados] autor[es] de un acto unilateral puede[n] invocar el error como vicio de la manifestación de voluntad si [dicho] acto ha sido formulado sobre la base de un error de hecho o de una situación cuya existencia diera por supuesta ese Estado en el momento de su formulación y ese hecho o esa situación constituyeran una base esencial de [la manifestación de voluntad] [consentimiento] para vincularse por tal acto. Lo anterior no se aplicará si el Estado autor [o los Estados autores] 
contibuyó [eron] con su conducta al error o si las circunstancias fueran tales que hubiera[n] quedado advertido de la posibilidad del error.

2. Dolo: El Estado [o los Estados] autor[es] de un acto unilateral puede[n] invocar el dolo como vicio de la manifestación de voluntad si ha[n] sido inducido[s] a formular dicho acto por la conducta fraudulenta de otro Estado.

3. Corrupción del representante de un Estado: El Estado [o los Estados] autor[es] de un acto unilateral puede[n] invocar un vicio de la manifestación de voluntad si dicho acto ha sido formulado mediante la corrupción de la persona que lo realiza, efectuada directa o indirectamente por otro Estado.

4. Coerción ejercida sobre la persona que realiza el acto: El Estado [o los Estados] autor[es] de un acto unilateral puede[n] invocar la nulidad absoluta si en la formulación del acto ha mediado la coerción sobre la persona que lo realiza, mediante acto o amenazas dirigidos contra ella.

5. Coerción por la amenaza o el uso de la fuerza: El Estado [o los Estados] autor[es] de un acto unilateral puede[n] invocar la nulidad absoluta de dicho acto, si su formulación ha sido obtenida por la amenaza o el uso de la fuerza en violación de los principios de derecho internacional incorporados en la Carta de las Naciones Unidas.

6. Acto unilateral contrario a una norma imperativa de derecho internacional (jus cogens): Un Estado podrá invocar la nulidad absoluta de un acto unilateral formulado por uno o varios Estados si dicho acto unilateral, en el momento de su formulación, está en oposición con una norma imperativa de derecho internacional.

7. Acto unilateral contrario a una decisión del Consejo de Seguridad: Un Estado podrá invocar la nulidad absoluta de un acto unilateral formulado por uno o varios Estados si dicho acto unilateral, en el momento de su formulación, contradice una decisión del Consejo de Seguridad.

8. Acto unilateral contrario a una norma fundamental del derecho interno del Estado autor: El Estado [o los Estados] autor[es] del acto unilateral podrán invocar su nulidad si dicho acto es contrario a una norma de importancia fundamental del derecho interno del Estado autor del acto.

B. Del momento a partir del cual el acto unilateral produce sus efectos jurídicos

Artículo 6. Momento a partir del cual el acto unilateral produce sus efectos jurídicos.

C. Del respeto y la aplicación de los actos unilaterales

Artículo 7. Acta sunt servanda

Todo acto unilateral en vigor obliga al Estado o los Estados que lo formulan y debe ser cumplido de buena fe.

Artículo 8. De la irretroactividad de los actos unilaterales 
El acto unilateral es aplicable a los hechos o situaciones posteriores a su formulación, salvo que el Estado o los Estados autores de dicho acto hayan manifestado de cualquier manera una intención diferente.

Artículo 9. De la aplicación territorial de los actos unilaterales

El acto unilateral vincula al Estado que lo formula en relación con la totalidad de su territorio, salvo que una manifestación diferente se desprenda o conste de otro modo.

D. De la interpretación de los actos unilaterales

Artículo 10. Regla general de interpretación:

1. Un acto unilateral deberá interpretarse de buena fe conforme al sentido ordinario que se atribuya a los términos de la declaración en su contexto y a la luz de la intención del Estado que lo formula.

2. A los fines de la interpretación de un acto unilateral, el contexto comprende, además del texto, su preámbulo y anexos.

3. Además del contexto, habrá de tenerse en cuenta toda práctica ulterior seguida en aplicación del acto y toda norma pertinente del derecho internacional aplicable en la relación entre el Estado o los Estados autores y el Estado o los Estados destinatarios.

Artículo 11. Medios complementarios de interpretación

Se podrá acudir a medios de interpretación complementarios, en particular [a los trabajos preparatorios y] a las circunstancias de la formulación del acto para confirmar el sentido resultante de la aplicación del artículo $10 \mathrm{o}$ para determinar el sentido cuando la interpretación dada de conformidad con el artículo 10:

1. Deje ambiguo u oscuro el sentido; o

2. Conduzca a un resultado manifiestamente absurdo o irrazonable.

Parte II. Reglas aplicables a los actos unilaterales por los cuales el Estado asume obligaciones.

Artículo 12. De la revocación de los actos unilaterales.

Artículo 13. De la modificación de los actos unilaterales.

Artículo 14. De la terminación y de la suspensión de la aplicación de los actos unilaterales. 